\title{
A blood less primitive
}

Cord blood provides important clues for directing human pluripotent stem cells to become pre-hematopoietic stem cells.

It only takes a single stem cell to reestablish the entire blood cell population of an animal that has been depleted of working bone marrow. Hematopoietic stem cells (HSCs) are important as research tools and for treating disorders and cancers of the blood; but they are challenging to purify, and the generation of fully functional HSCs in vitro remains elusive. Andrew Elefanty, Elizabeth Ng and Ed Stanley at the Murdoch Childrens Research Institute in Melbourne, Australia, have used insights from the young embryo to generate cells in culture that more closely resemble immature human HSCs.

Blood cells first appear in the yolk sac, but this 'primitive' hematopoiesis does not generate repopulating stem cells. A second wave yields multiple hematopoietic lineages that still lack HSCs capable of blood repopulation. True HSCs first arise from endothelial cells that line the embryonic aorta in a region of the embryo known as the aorta-gonadmesonephros (AGM).

Researchers in the blood stem cell field have largely worked with primitive hematopoietic cells generated from pluripotent stem cells. Part of the reason for this is practical, since the earliest blood in differentiating pluripotent stem cell cultures is primitive- "It's the first thing you'll see," says Ng. These cells express many HSC genes and home correctly to bone marrow, but they do not engraft. The Australian team came to believe that the cells don't just represent a hurdle to deriving true 'definitive' HSCs, but a blind alley. "They're the wrong cells," says Elefanty. "The field now realizes that those populations are not interchangeable."

In the hopes of identifying AGM-like cells in culture, the scientists first generated human embryonic stem cell lines bearing fluorescent markers for RUNX1C, which is expressed in repopulating HSCs from human AGM, and for SOX17, an arterial marker required for AGM hematopoiesis in mouse. Using their protocol to generate highly consistent pluripotent cell aggregates, known as 'spin embryoid bodies', and a defined medium for well-controlled hematopoietic differentiation, they generated colony-forming cells that home to bone marrow but do not engraft.

Next, they compared their fluorescencesorted RUNX1C-expressing cells with cells that can engraft from cord blood. Of the over 30 genes with higher expression in engrafting cells, three HOXA genes stood out. Misexpression of HOX genes is associated with aberrant hematopoiesis and leukemia, and "those genes influence cell fate and axial position along the embryo and they're expressed very early on, right at the onset of mesoderm commitment," says Elefanty.

The results hinted that cells need to be correctly patterned at the time of mesoderm differentiation to form definitive HSCs. Previous work implicated Wnt and Activin signaling in this specification event, and the researchers ultimately found that a twoday pulse of a Wnt agonist and an Activin antagonist generated large, distinctive multifocal colonies of erythroid and myeloid cells. Untreated cultures would produce plenty of blood but die out in two weeks, whereas their correctly patterned blood cells lasted beyond five weeks. "That's a significant property of cord blood, that it keeps growing," says Stanley.

Unlike controls, treated cells formed vascular structures that express SOX17. RUNX1C-expressing hematopoietic cells appeared within these structures, and some cells expressed both markers, recapitulating endothelial-to-blood transitions that occur in the AGM. Remarkably, treated cells showed a dramatic switch in globin profile from fetal to adult isoforms.

The correctly patterned cells, however, cannot repopulate the blood. RNA sequencing of the RUNX1C-expressing population indicated that these cells resemble 'preHSCs' in the AGM, which must mature

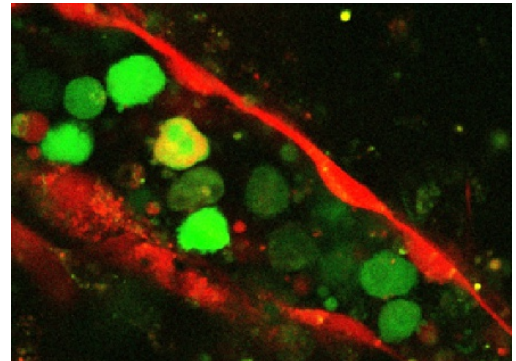

A new protocol generates blood-producing vasculature (marked by SOX17 expression, red) and pre-hematopoiesis stem cells (marked by RUNX1C, green).

during their migration to the fetal liver before they gain the ability to repopulate. Comparison with sorted cells from human AGM and fetal liver samples revealed some gene expression differences, notably that retinoic acid signaling is more active in the in vivo cells. The researchers are actively exploring retinoic acid signaling as a way of maturing cells in culture.

The new work emphasizes that tricking cells into believing that they are at a particular time and place in development, despite the simpler and sped-up environment of the dish, is key. "If you haven't made mesoderm with the right $H O X$ code, then you're in the wrong part of the developing human," says $\mathrm{Ng}$. "A lot of our work is now focused on getting the $H O X$ code in our cells to match up with things we know occur in the embryo," adds Stanley. Elefanty believes that the need for correct $H O X$ signatures is a lesson that generalizes across differentiation contexts. With AGM-like hematopoiesis now in hand, the three researchers, who have brought complementary skills and a knack for frank discussion into a productive collaboration of over 15 years, have a promising path for producing mature HSCs.

Tal Nawy

\section{RESEARCH PAPERS}

$\mathrm{Ng}, \mathrm{E}$. S. et al. Differentiation of human embryonic stem cells to $\mathrm{HOXA}^{+}$hemogenic vasculature that resembles the aorta-gonad-mesonephros. Nat. Biotechnol. 34, 1168-1179 (2016). 